\title{
THE ROLE OF BIOMARKERS IN IDENTIFYING HYPOXIC ISCHAEMIC ENCEPHALOPATHY IN NEONATES AND PREDICTING LONG TERM NEURODEVELOPMENTAL OUTCOMES - A LITERATURE REVIEW
}

\author{
Niall Dunworth ${ }^{1}$, Niazy Al Assaf², Rizwan Khan ${ }^{2}$ \\ 1 Graduate Entry Medical School, University of Limerick \\ 2 Department of Neonatology, University Maternity Hospital Limerick
}

\section{Introduction and Aims}

Currently, identification of Hypoxic Ischaemic Encephalopathy (HIE) relies on clinical signs and diagnostic imaging. These modalities have limitations, prompting the identification of specific biomarkers which are elevated in HIE, thus identifying and risk stratifying HIE patients. This literature review aims to:

- Review recent work on biomarkers which are associated with HIE

- Determine which biomarkers predict neurodevelopmental outcome

\section{Methods}

A systematic review was conducted using articles from: Pubmed, Embase, Medline, Web of Science, ScienceDirect

Search terms included: Hypoxic ischaemic encephalopathy, predictors of outcome, biomarkers and HIE, cord blood biomarkers and HIE.

Inclusion criteria: Studies from 2010 - present.

Exclusion criteria: Studies performed on animal subjects.

\section{Results}

\section{Escobar et al 2010}

UCHL1 levels higher in HIE patients than controls

Ennen et al 2011

- $\quad$ GFAP levels higher in moderate/severe HIE patients for the first week of life compared to non HIE controls

Massaro et al 2013

- UCHL1 higher at 0 and 72 hours, GFAP levels higher at 24 and 72 hours in infants with severe HIE signs on MR

Both superior predictors of outcome compared to EEG \& APGAR

Chalak et al 2015

- $\quad$ Serum UCHL1 increased initially but drops within 6 hours, serum GFAP raises over time in HIE infants receiving therapeutic hypothermia(figures $1 \& 2$ )

GFAP levels higher in infants with abnormal neurological outcome at 6-24 months

Escobar et al 2014

- UCHL1 elevated at 0-6 hours but dropped by 24 hours in HIE

GFAP levels rose over 96 hours (figures $3 \& 4$ )

UCHL1 levels correlated with developmental outcome at 8 months

Looney et al 2015

- No significant difference in umbilical cord blood (UCB) GFAP levels in HIE and non HIE controls

- No difference in UCB GFAP in mild, moderate and severe HIE or between those with normal or abnormal developmental outcome

Zaigham et al 2015

- No significant difference in cord blood UCHL1 and GFAP levels between grades II and III HIE

No difference in levels between patients with or without sequalae at follow-up

Roka et al 2011

- $\quad$ S100ß and NSE lower in in healthier infants;

- $\quad \mathrm{S} 100 \beta$ lower in group receiving Therapeutic Hypothermia and correlates with neurodevelopmental outcome

\section{Results}

Massaro et al 2014

- $\quad$ Elevated S100B and NSE during therapeutic hypothermia associated with poorer neurodevelopmental outcomes

Gazollo et al 2015

- $\quad$ Salivary S100 $\beta$ higher in infants with poor outcomes at 12 months following perinatal asphyxia

Chaparro- Huerta et al 2017

- $\quad$ Serum S100ß and NSE higher in infants with perinatal asphyxia compared to healthy controls

Karlsson et al 2010

- $\quad$ LDH levels predict long term outcome after HIE

Hayakawa et al 2014

- $\quad$ Serum Lactate and LDH higher in infants with poor outcome

Mehta et al 2015

- $\quad$ Salivary \& serum LDH increased with presence and severity of HIE and in infants with poor neurological outcome

Chiang et al 2016

- $\quad$ Serum lactate higher at 72 hours post hypothermia in infants found to have poor neurodevelopmental outcomes at 24 months
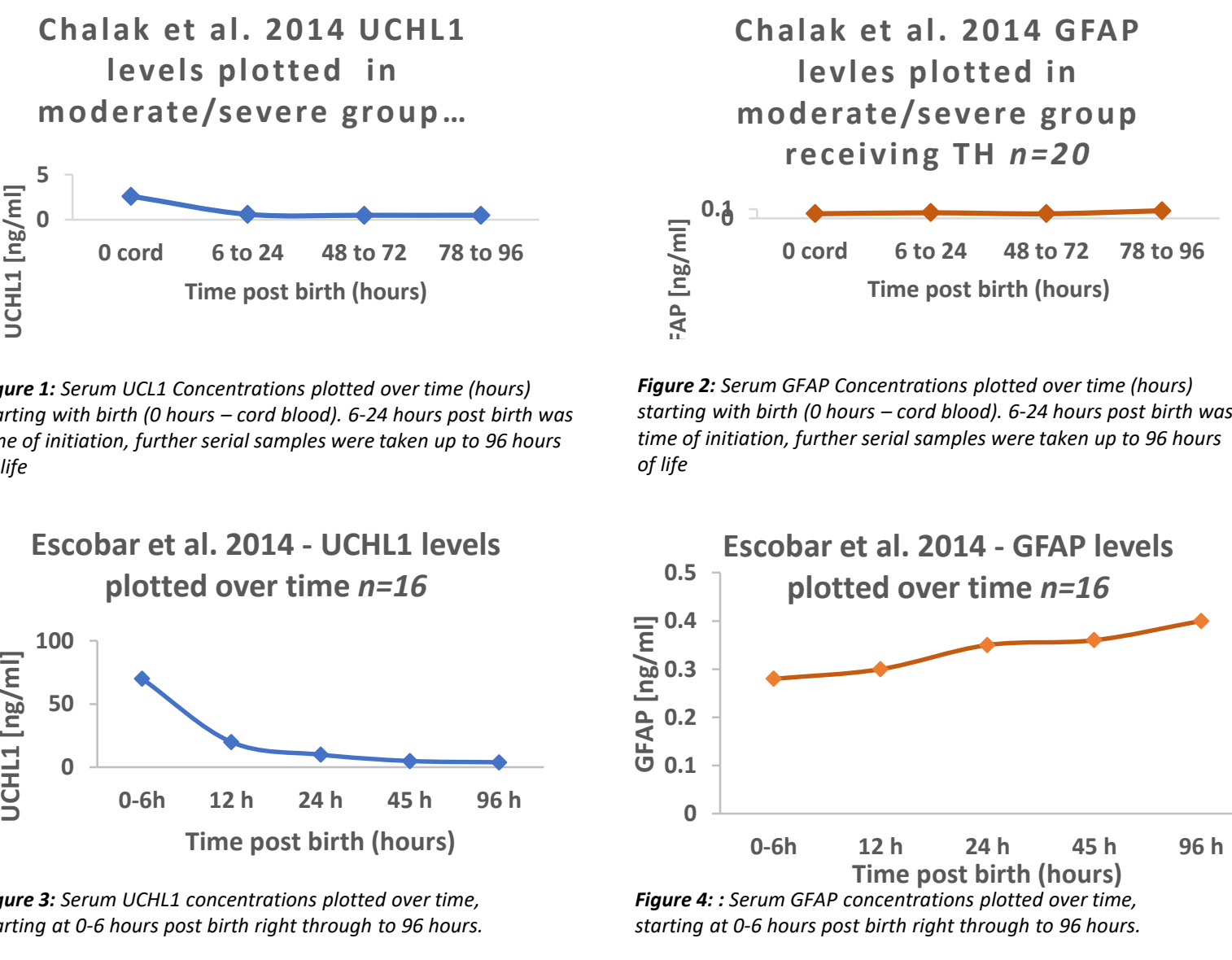

\section{Conclusion}

Serum UCHL1 and GFAP levels increase in the presence of HIE UCHL1 is raised at an early stage but reduced by 24 hours, GFAP is lower initially but increased with time

UCHL1 and GFAP concentrations correlate with severity of HIE and poor neurodevelopmental outcome

- $\quad$ Studies focusing only on umbilical cord GFAP and UCLH1 show no correlation with the presence or severity of HIE or with neurodevelopmental outcome. NSE levels correlate with the presence of HIE

S100 $\beta$ correlates with the presence and increased severity of HIE and with poor neurodevelopmental outcome

Serum lactate, serum LDH and salivary LDH correlate with increased severity of HIE and poor neurodevelopmental outcomes 